\title{
Scattering due to geometry: Case of a spinless particle moving on an asymptotically flat embedded surface
}

\author{
Neslihan Oflaz, ${ }^{1}$ Ali Mostafazadeh, ${ }^{1}$ and Mehrdad Ahmady ${ }^{2}$ \\ ${ }^{1}$ Departments of Mathematics and Physics, Koç University, 34450 Sartyer, Istanbul, Turkey \\ ${ }^{2}$ Department of Physics, Azarbaijan University of Shahid Madani, 53714-161 Tabriz, Iran
}

(Received 9 January 2018; published 20 August 2018)

\begin{abstract}
A nonrelativistic quantum mechanical particle moving freely on a curved surface feels the effect of the nontrivial geometry of the surface through the kinetic part of the Hamiltonian, which is proportional to the Laplace-Beltrami operator, and a geometric potential, which is a linear combination of the mean and Gaussian curvatures of the surface. The coefficients of these terms cannot be uniquely determined by general principles of quantum mechanics but enter the calculation of various physical quantities. We examine their contribution to the geometric scattering of a scalar particle moving on an asymptotically flat embedded surface. In particular, having in mind the possibility of an experimental realization of the geometric scattering in a low-density electron gas formed on a bumped surface, we determine the scattering amplitude for arbitrary choices of the curvature coefficients for a surface with global or local cylindrical symmetry. We also examine the effect of perturbations that violate this symmetry and consider surfaces involving bumps that form a lattice.
\end{abstract}

DOI: 10.1103/PhysRevA.98.022126

\section{INTRODUCTION}

The study of quantum mechanics of nonrelativisitic particles moving in a curved Riemannian manifold has been a focus of attention since the early days of canonical quantum gravity. In his pioneering works of the 1950s, DeWitt explored the consequences of the form invariance of the Hamiltonian under the group of general point transformations of the configuration space [1] and discovered the surprising fact that the path-integral quantization of a scalar particle moving in a Riemannian manifold $\mathcal{M}$ leads to a quantum Hamiltonian operator $H$ that besides the expected kinetic term, which is proportional to the Laplace-Beltrami operator, included a term of the form $\hbar^{2} R / 12 m$, where $R$ is the Ricci scalar curvature of the manifold [2]. See also Refs. [3,4].

The fact that the curvature term is proportional to $\hbar^{2}$ is a clear indication that it is a by-product of the quantization of the associated classical system. The latter is defined by a classical Hamiltonian of the form

$$
H_{c}=\frac{1}{2 m} g^{i j} p_{i} p_{j},
$$

where $g^{i j}$ are the coefficient of the inverse of the metric tensor $\mathbf{g}=\left(g_{i j}\right)$ in a local coordinate frame, and Einstein's summation convention is employed.

In the canonical quantization program, the coefficient of the curvature term is related to the choice of ordering of factors in the quantum analog of (1). Indeed, different factorordering prescriptions that yield a scalar Hamiltonian operator $H$ correspond to different choices for the coefficient of the curvature term; in general,

$$
H=-\frac{\hbar^{2}}{2 m} \Delta_{g}+\frac{\lambda \hbar^{2}}{m} R,
$$

where $\Delta_{g}$ is the Laplace-Beltrami operator for the metric tensor g, i.e., the operator acting on the scalar functions $\psi: \mathcal{M} \rightarrow \mathbb{C}$ according to

$$
\left(\Delta_{g} \psi\right)(x):=g^{-1 / 2} \partial_{i}\left[g^{i j} g^{1 / 2} \partial_{j} \psi(x)\right],
$$

$g:=\operatorname{det}(\mathbf{g})$, and $\lambda$ is a real coefficient whose choice cannot be fixed using basic principles of quantum mechanics.

In the path-integral quantization scheme, the coefficient of the curvature term turns out to depend on the choice of the path integral measure [5]. For example, in Ref. [6] DeWitt uses a different choice of the measure that corresponds to $\lambda=1 / 8$ (rather than $\lambda=1 / 12$ of [2].) See also Ref. [7]. The choice $\lambda=1 / 8$ turns out to be consistent with the result obtained by taking the bosonic part of a supersymmetric quantum Hamiltonian used in the path-integral proofs of the Atiyah-Singer index theorem $[8,9]$ where supersymmetry removes the factorordering ambiguity. Other choices have also been considered and promoted in the literature. For example, Refs. [10,11] show that the requirement of conformal invariance of $H$ corresponds to taking $\lambda=(n-1) / 8(n-1)$, where $n$ is the dimension of $M$. For $n=2$, this gives $\lambda=1 / 8$. References [12,13] present arguments supporting the choice $\lambda=0$. The review article [14] provides a summary of the related developments up to the year 1980.

The author of Ref. [5] points out that the ambiguity related to the choice of $\lambda$ could be settled only using the experimental data obtained for the particular system in question. This point of view was adopted independently in Ref. [15] where a first step in this direction was taken by computing the effect of the scalar curvature term $\lambda \hbar^{2} R / m$ on the scattering cross section of a particle moving in a cylindrically symmetric asymptotically flat surface. Here the basic idea is to determine the dependence of the scattering data on the value of $\lambda$ and try to pave the way for fixing this value by comparing the theoretical results with the outcome of a suitable scattering experiment. This is 
done by writing the Hamiltonian operator (2) as the sum of the Hamiltonian operator:

$$
H_{0}:=-\frac{\hbar^{2}}{2 m} \nabla^{2},
$$

for a free particle moving in a plane and an effective scattering potential:

$$
V:=H-H_{0} .
$$

The latter is then treated as a perturbation, and the machinery of the first-order Born approximation is used to compute the scattering amplitude and the cross section for $V$.

The preliminary results reported in Ref. [15] indicate that the scattering effect due to the geometry of a Gaussian bump is actually not unrealistically small. However, there is a basic difficulty with the experimental realization of these results because any realistic experimental setup that aims at probing such an scattering effect would involve particles whose motion is constrained to take place in a surface by certain constraining forces that act in the three-dimensional Euclidean space $\mathbb{E}^{3}$. In other words, the configuration space of the particle is an embedded surface. It is well known that the classical mechanical system involves only the metric of the surface that is induced by its embedding in $\mathbb{E}^{3}$. This does not carry any information about the details of how the surface is embedded in $\mathbb{E}^{3}$, i.e., it is sensitive only to the intrinsic geometry of the surface. It is remarkable that the same does not seem to hold for a quantum particle; a quantum particle would know about the extrinsic geometry of the surface as well.

The study of the quantum mechanics of a particle constrained to move in a manifold embedded in a Euclidean space has a long history. There are two different approaches for dealing with this problem: Dirac's formulation of constrained Hamiltonian systems [16] and the thin-layer quantization scheme developed in [17-24].

The application of Dirac's formulation to a particle constrained to move on a surface $S$ embedded in $\mathbb{E}^{3}$ yields second class constraints whose details depend on the choice of the equation used to characterize $S$. As different equations can describe the same embedded surface, Dirac's method turns out to be ambiguous [25]. See also Ref. [26].

The thin-layer method as outlined in Ref. [18] involves three steps. First, one considers a particle that is free to move in a thin layer parallel to the embedded surface. Second, one carries out a careful decoupling of the motion along the tangential and normal directions to the embedded surface. Third, one uses a careful limiting process that essentially removes the information about the motion along the normal direction and yields a Schrödinger equation in the tangential coordinates and a corresponding effective Hamiltonian. This amounts to assuming that the particle is in the ground state of a barrier potential that keeps it in the vicinity of the surface along the normal direction [23]. The Hamiltonian obtained by the thin layer method has the form $[18,19,23]$

$$
H=-\frac{\hbar^{2}}{2 m} \Delta_{g}+\frac{\hbar^{2}}{2 m}\left(K-M^{2}\right),
$$

where $M$ and $K=R / 2$ are, respectively, the mean and Gaussian curvatures of the surface $S$ [27]. Unlike the Gaussian curvature, which is uniquely determined by the metric tensor of $S$, the mean curvature is sensitive to the way $S$ is embedded in $\mathbb{E}^{3}$, i.e., it is a measure of the extrinsic geometry of $S$. Similar results have also been obtained within the context of Dirac's method for particular choices of the constraint equation that specify $S$ [28]. The authors of Ref. [29] show that the choice of the constraining forces which in practice have a finite strength can lead to the addition of a term proportional to $\hbar^{2}$ to the geometric potential. Therefore, similarly to the Dirac's method, the thin-layer quantization scheme that involves realistic constraining forces also suffers from ambiguities in the choice of the Hamiltonian operator.

To the best of our knowledge, the only experimental study of the predictions of the thin-layer quantization method is the one reported in Ref. [30], where the authors consider the effect of the geometric potential on the electronic properties of certain liquids. The physical implications of the geometric potentials have also been studied in Refs. [31-35].

The purpose of the present article is to use the approach of Ref. [15] to explore the phenomenon of geometric scattering for an asymptotically Euclidean embedded surface $S$. Specifically, we consider the geometric scattering of a scalar particle of mass $m$ whose motion in $S$ is described by the Hamiltonian operator:

$$
H=-\frac{\hbar^{2}}{2 m} \Delta_{g}+\frac{\hbar^{2}}{m}\left(\lambda_{1} K+\lambda_{2} M^{2}\right),
$$

where $\lambda_{1}$ and $\lambda_{2}$ are arbitrary real coefficients.

\section{GEOMETRIC SCATTERING AMPLITUDE}

We begin our analysis by recalling the LippmannSchwinger equation for a Hamiltonian of the form $H=H_{0}+V$,

$$
\left|\psi^{( \pm)}\right\rangle=|\phi\rangle+\frac{1}{E-H_{0} \pm i \epsilon} V\left|\psi^{( \pm)}\right\rangle,
$$

where we use the notation of Ref. [36]. In particular, $H_{0}$ is the free Hamiltonian, and $|\phi\rangle$ and $E$ are, respectively, the state vector and the energy of the incident particle that satisfy $H_{0}|\phi\rangle=E|\phi\rangle$. The two-dimensional scattering problem for the interaction potential $V$ consists of computing the scattering amplitude $f\left(\vec{k}^{\prime}, \vec{k}\right)$, which is related to $\left|\psi^{(+)}\right\rangle$according to

$$
\left\langle\vec{x} \mid \psi^{(+)}\right\rangle=\frac{1}{2 \pi}\left[e^{i \vec{k} \cdot \vec{x}}+\frac{e^{i k r}}{\sqrt{r}} f\left(\vec{k}^{\prime}, \vec{k}\right)\right] .
$$

Here $\vec{x}=\left(x^{1}:=x, x^{2}:=y\right)$ marks the Cartesian coordinates in $\mathbb{R}^{2}$, and $\vec{k}$ and $\vec{k}^{\prime}$ are, respectively, the wave vector for the incident and scattered wave functions, $k:=|\vec{k}|=\sqrt{2 m E} / \hbar$, $r:=|\vec{x}|$, and $\vec{k}^{\prime}=k \vec{x} / r$.

We can express the scattering amplitude in terms of the interaction potential via

$$
f\left(\vec{k}^{\prime}, \vec{k}\right)=\frac{\sqrt{2 \pi} m e^{-3 i \pi / 4}}{\sqrt{k} \hbar^{2}} \int d^{2} \vec{x}^{\prime} e^{-i \vec{k}^{\prime} \cdot \vec{x}^{\prime}}\left\langle\vec{x}^{\prime}|\hat{V}| \psi^{(+)}\right\rangle,
$$

and compute the differential cross section using

$$
\frac{d \sigma\left(\vec{k}^{\prime}, \vec{k}\right)}{d \Omega}=\left|f\left(\vec{k}^{\prime}, \vec{k}\right)\right|^{2} .
$$

To perform the first Born approximation, we replace the $\left|\psi^{(+)}\right\rangle$ appearing on the right-hand side of (10) by the state vector $|\vec{k}\rangle$ 
for the incident particle. This gives

$$
\begin{aligned}
f\left(\vec{k}^{\prime}, \vec{k}\right) & \approx f^{(1)}\left(\vec{k}^{\prime}, \vec{k}\right) \\
& =\frac{-i \sqrt{2 \pi} m e^{-i \pi / 4}}{\sqrt{k} \hbar^{2}} \int d \vec{x}^{\prime 2} e^{-i \vec{k}^{\prime} \cdot \vec{x}^{\prime}}\left\langle\vec{x}^{\prime}|\hat{V}| \vec{k}\right\rangle .
\end{aligned}
$$

For the geometric scattering problem determined by the Hamiltonian operator (7), the free Hamiltonian $H_{0}$ and the interaction potential $V$ are, respectively, given by (4) and (5). These relations together with (7) imply

$$
\begin{aligned}
\left\langle\vec{x}^{\prime}|\hat{V}| \vec{k}\right\rangle= & \frac{\hbar^{2}}{4 \pi m}\left[\left(g_{0}^{i j}-g^{i j}\right) \partial_{i}^{\prime} \partial_{j}^{\prime}-\frac{\partial_{i}^{\prime}\left(\sqrt{g} g^{i j}\right)}{\sqrt{g}} \partial_{j}^{\prime}\right. \\
& \left.+2\left(\lambda_{1} K+\lambda_{2} M^{2}\right)\right] e^{i \vec{k} \cdot \vec{x}^{\prime}},
\end{aligned}
$$

where $g_{0}^{i j}$ stands for the components of the inverse of the Euclidean metric tensor (which coincides with the Kronecker delta symbol $\delta_{i j}$ when $x^{\prime i}$ label Cartesian coordinates), $\partial_{i}^{\prime}$ means partial derivation with respect to $x^{\prime i}$, the quantities $g_{0}^{i j}$, $g^{i j}, g, K$, and $M$ are evaluated at $\vec{x}^{\prime}$, and we have employed $\left\langle\vec{x}^{\prime} \mid \vec{k}\right\rangle=e^{i \vec{k} \cdot \vec{x}^{\prime}} / 2 \pi$.

\section{GEOMETRIC SCATTERING FOR A CYLINDRICALLY SYMMETRIC SURFACE}

Suppose that the surface $S$ is the graph of a smooth function of the radial coordinate $r$ in the polar coordinate system in $\mathbb{R}^{2}$, i.e., there is a smooth function $f:[0, \infty) \rightarrow \mathbb{R}$ such that

$$
z=f(r),
$$

where $(r, \theta, z)$ are cylindrical coordinates on $\mathbb{R}^{3}$. This equation determines a smooth embedded surface provided that it has a vanishing derivative at $r=0$, that is, $\dot{f}(0)=0$, where an overdot means derivation with respect to $r$.

We can identify $(r, \theta)$ with the polar coordinates and express the metric tensor induced from the Euclidean geometry of $\mathbb{E}^{3}$ on $S$ in these coordinates as

$$
\left[g_{i j}\right]=\left[\begin{array}{cc}
1+\dot{f}^{2} & 0 \\
0 & r^{2}
\end{array}\right] .
$$

Here the values 1 and 2 of the coordinate labels $i$ and $j$ correspond to $r$ and $\theta$, respectively. In view of (15), the Gaussian and mean curvatures of $S$ are, respectively, given by

$$
K=\frac{G \dot{G}}{r}, \quad M=\frac{1}{2}\left(\frac{G}{r}+\dot{G}\right)
$$

where

$$
G:=\frac{\dot{f}}{\sqrt{1+\dot{f}^{2}}} .
$$

According to (16) and (17), $K$ and $M$ are regular (nonsingular) functions of $r$ provided that $\dot{f}(r) / r$ tend to a finite limit as $r \rightarrow 0$. In what follows we assume that this condition holds.

In view of (13), (15), and (16),

$$
\left\langle x^{\prime}|\hat{V}| k\right\rangle=\frac{\hbar^{2}}{4 \pi m}\left\{-G^{2}\left(\frac{\vec{k} \cdot \vec{x}^{\prime}}{r^{\prime 2}}\right)^{2}+i\left[\frac{1}{r^{\prime}} G^{2}+G \dot{G}\right]\left(\frac{\vec{k} \cdot \vec{x}^{\prime}}{r^{\prime}}\right)+2 \lambda_{1} \frac{G \dot{G}}{r^{\prime}}+\frac{\lambda_{2}}{2}\left(\frac{G^{2}}{r^{2}}+2 \frac{G \dot{G}}{r}+\dot{G}^{2}\right)\right\} e^{i \vec{k} \cdot \vec{x}^{\prime}} .
$$

Substituting this relation in (12), we find

$$
f^{(1)}\left(\vec{k}^{\prime}, \vec{k}\right)=\frac{e^{-3 \pi i / 4}}{\sqrt{8 \pi k}} \int d^{2} \vec{x}^{\prime} e^{i\left(\vec{k}-\vec{k}^{\prime}\right) \cdot \vec{x}^{\prime}}\left\{-G^{2}\left(\frac{\vec{k} \cdot \vec{x}^{\prime}}{r^{\prime}}\right)^{2}+i\left[\frac{1}{r^{\prime}} G^{2}+G \dot{G}\right]\left(\frac{\vec{k} \cdot \vec{x}^{\prime}}{r^{\prime}}\right)+2 \lambda_{1} \frac{G \dot{G}}{r^{\prime}}+\frac{\lambda_{2}}{2}\left(\frac{G^{2}}{r^{2}}+2 \frac{G \dot{G}}{r}+\dot{G}^{2}\right)\right\} e^{i \vec{k} \cdot \vec{x}^{\prime}} .
$$

In order to evaluate the integral in this equation, we work in a Cartesian coordinate system $\left(x^{\prime}, y^{\prime}\right)$ where $\Delta \vec{k}:=\vec{k}-\vec{k}^{\prime}$ is along the $x^{\prime}$ axis. Transforming to the corresponding polar coordinates $\left(r^{\prime}, \theta^{\prime}\right)$ we can perform the integral over $\theta^{\prime}$. This gives

$$
\begin{aligned}
f^{(1)}\left(\vec{k}^{\prime}, \vec{k}\right)= & \sqrt{\frac{\pi}{2 k}} e^{-3 \pi i / 4} \int_{0}^{\infty} d r^{\prime}\left\{\left[-r^{\prime} G^{2} k_{x}^{2}+2 \lambda_{1} G \dot{G}+\frac{\lambda_{2}}{2}\left(\frac{G^{2}}{r}+2 G \dot{G}+r \dot{G}^{2}\right)\right] J_{0}\left(r^{\prime}|\Delta \vec{k}|\right)\right. \\
& \left.+\left[-G^{2} \frac{k_{y}^{2}-k_{x}^{2}}{|\Delta \vec{k}|}-k_{x}\left(G^{2}+r^{\prime} G \dot{G}\right)\right] J_{1}\left(r^{\prime}|\Delta \vec{k}|\right)\right\},
\end{aligned}
$$

where $J_{0}$ and $J_{1}$ are the Bessel functions of the first kind. Denoting the angle between $\vec{k}$ and $\vec{k}^{\prime}$ by $\Theta$, and recalling that $\overrightarrow{k^{\prime}}=k \vec{x} /|\vec{x}|$, we have

$$
|\Delta \vec{k}|=2 k \sin \left(\frac{\Theta}{2}\right)=2 k_{x}, \quad k_{y}^{2}-k_{x}^{2}=k^{2} \cos \Theta .
$$

With the help of these relations, we can write (19) in the form

$$
\begin{aligned}
f^{(1)}\left(\vec{k}^{\prime}, \vec{k}\right)= & \sqrt{\frac{\pi}{2 k}} e^{-3 \pi i / 4} \int_{0}^{\infty} d r\left\{\left[-k^{2} r \sin ^{2}\left(\frac{\Theta}{2}\right) G^{2}+2 \lambda_{1} G \dot{G}+\frac{\lambda_{2}}{2}\left(\frac{G^{2}}{r}+2 G \dot{G}+r \dot{G}^{2}\right)\right] J_{0}\left(2 k r \sin \frac{\Theta}{2}\right)\right. \\
& \left.+\left[-\frac{k G^{2}}{2 \sin \frac{\Theta}{2}}-k r \sin \left(\frac{\Theta}{2}\right) G \dot{G}\right] J_{1}\left(2 k r \sin \frac{\Theta}{2}\right)\right\} .
\end{aligned}
$$


Next, suppose that

$$
\lim _{r \rightarrow \infty} r J_{1}\left(2 k r \sin \frac{\Theta}{2}\right) G(r)^{2}=0
$$

which roughly speaking means that as $r \rightarrow \infty,|G(r)|$ tends to 0 faster than $r^{-1 / 4}$. This is indeed a rather weak condition that we can easily fulfill for concrete applications. In view of (21), the fact that

$$
\lim _{r \rightarrow 0} \dot{f}(r)=\lim _{r \rightarrow \infty} \dot{f}(r)=0,
$$

and various properties of the Bessel functions, we have managed to express (20) in the form

$$
\begin{aligned}
f^{(1)}\left(\vec{k}^{\prime}, \vec{k}\right) & \\
= & \sqrt{\frac{\pi}{2 k}} e^{-3 \pi i / 4} \int_{0}^{\infty} d r\left[\frac{\lambda_{2}}{2}\left(\frac{G^{2}}{r}+r \dot{G}^{2}\right)\right. \\
& \times J_{0}\left(2 k r \sin \frac{\Theta}{2}\right)+k \sin \left(\frac{\Theta}{2}\right) G^{2} \\
& \left.\times\left(-\frac{1}{2 \sin ^{2} \Theta / 2}+2 \lambda_{1}+\lambda_{2}\right) J_{1}\left(2 k r \sin \frac{\Theta}{2}\right)\right] .
\end{aligned}
$$

For the forward scattering $(\Theta=0)$, this equation reduces to

$$
\begin{aligned}
f^{(1)}(\vec{k}, \vec{k})= & \sqrt{\frac{\pi}{2 k}} e^{-3 \pi i / 4} \int_{0}^{\infty} d r \\
& \times\left[\frac{\lambda_{2}}{2}\left(\frac{G^{2}}{r}+r \dot{G}^{2}\right)-\frac{k^{2}}{2} r G^{2}\right] .
\end{aligned}
$$

In particular, the Gaussian curvature of the surface does not affect the forward scattering amplitude. In contrast the scattering amplitude for backscattering $(\Theta=\pi)$ depends on both mean and Gaussian curvatures of the surface:

$$
\begin{aligned}
& f^{(1)}\left(\vec{k}^{\prime}=-\vec{k}, \vec{k}\right) \\
& =\sqrt{\frac{\pi}{2 k}} e^{-3 \pi i / 4} \int_{0}^{\infty} d r\left[\frac{\lambda_{2}}{2}\left(\frac{G^{2}}{r}+r \dot{G}^{2}\right) J_{0}(2 k r)\right. \\
& \left.\quad+k\left(2 \lambda_{1}+\lambda_{2}-\frac{1}{2}\right) G^{2} J_{1}(2 k r)\right] .
\end{aligned}
$$

As an example, consider a surface $S$ that has the shape of a Gaussian bump. Specifically, $S$ is given by (14) and

$$
f(r)=\delta e^{-r^{2} / 2 \sigma^{2}}
$$

where $\delta$ and $\sigma$ are real parameters. It is easy to see that this choice for $f(x)$ complies with the condition (21). Therefore, Eqs. (22)-(24) hold. Let us introduce the dimensionless parameter

$$
\eta:=\left(\frac{\delta}{\sigma}\right)^{2}
$$

and compute the scattering amplitude (22) as a power series in $\eta$. This gives

$$
\begin{aligned}
f^{(1)}\left(\vec{k}^{\prime}, \vec{k}\right)= & \sqrt{\frac{\pi}{2 k}} e^{-3 \pi i / 4}\left[\sigma^{2} k^{2}\left(\lambda_{1} \sin ^{2} \frac{\Theta}{2}-\frac{1}{4}\right)\right. \\
& \left.+\frac{\lambda_{2}}{4}\left(\sigma^{4} k^{4} \sin ^{4} \frac{\Theta}{2}+2\right)\right] \\
& \times \exp \left(-\sigma^{2} k^{2} \sin ^{2} \frac{\Theta}{2}\right) \eta+O\left(\eta^{2}\right),
\end{aligned}
$$

where $O\left(\eta^{\ell}\right)$ stands for the terms of order $\ell$ and higher in powers of $\eta$. For $|\eta| \ll 1$ we can safely ignore $O\left(\eta^{2}\right)$ if $k$ is of the order of $\sigma^{-1}$ or smaller. In particular, for $\vec{k}^{\prime}= \pm \vec{k}$, we have

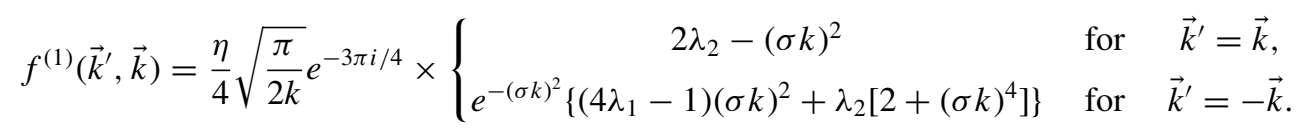

This equation shows that we should be able to determine the coefficients $\lambda_{1}$ and $\lambda_{2}$ by examining the forward and backward scattering data for incident particles with different values of $k$. Figure 1 shows the plots of the differential scattering cross section $\left|f^{(1)}\left(\vec{k}^{\prime}, \vec{k}\right)\right|^{2}$ for $\Theta=0, \frac{\pi}{6}, \frac{\pi}{4}, \pi$ and the choice $\lambda_{1}=-\lambda_{2}=\frac{1}{2}$ that is obtained in the thin-layer quantization scheme [18]. According to this figure, there is a basic difference between the forward and nonforward scattering cross sections. For $\Theta \neq 0$, the differential cross section has a peak that decreases in hight and shifts to the left as we increase $\Theta$.

We can also compute the total scattering cross section to leading order in $\eta$. The result is

$$
\begin{aligned}
\sigma_{\text {tot. }}^{(1)}= & \int_{0}^{2 \pi}\left|f^{(1)}\left(\vec{k}^{\prime}, \vec{k}\right)\right|^{2} d \theta=\frac{\pi^{2}}{256 k} e^{-\sigma^{2} k^{2}}\left[\mathfrak{p}_{0}\left(\sigma^{2} k^{2}\right)\right. \\
& \left.\times I_{0}\left(\sigma^{2} k^{2}\right)+\mathfrak{p}_{1}\left(\sigma^{2} k^{2}\right) I_{1}\left(\sigma^{2} k^{2}\right)\right] \eta^{2}+O\left(\eta^{3}\right),
\end{aligned}
$$

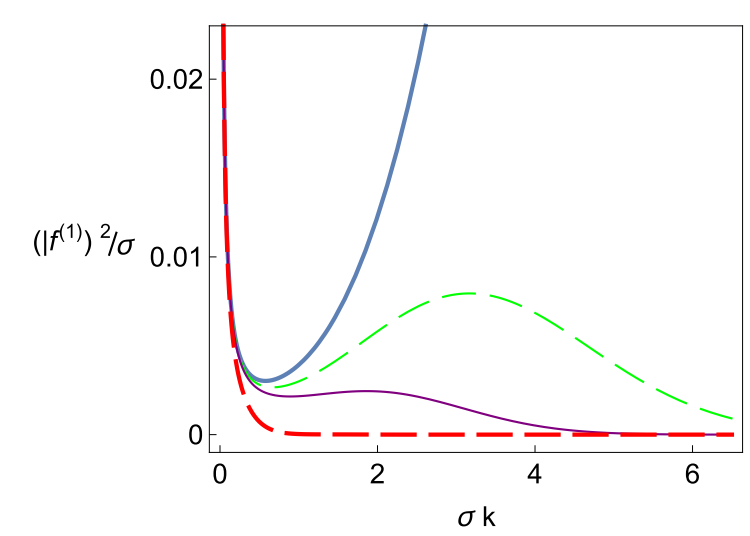

FIG. 1. Plots of $\left|f^{(1)}\right|^{2} / \sigma$ as a function of $\sigma k$ for $\Theta=0$, i.e., forward scattering (thick solid blue curve), $\Theta=\pi / 6$ (thin dashed green curve), and $\Theta=\pi / 4$ (thin solid purple curve), and $\Theta=\pi$, i.e., backward scattering (thick dashed red curve) for a Gaussian bump (25) with $\eta=0.1$. Here we have taken $\lambda_{1}=-\lambda_{2}=\frac{1}{2}$, which follows from the thin-layer quantization scheme. 


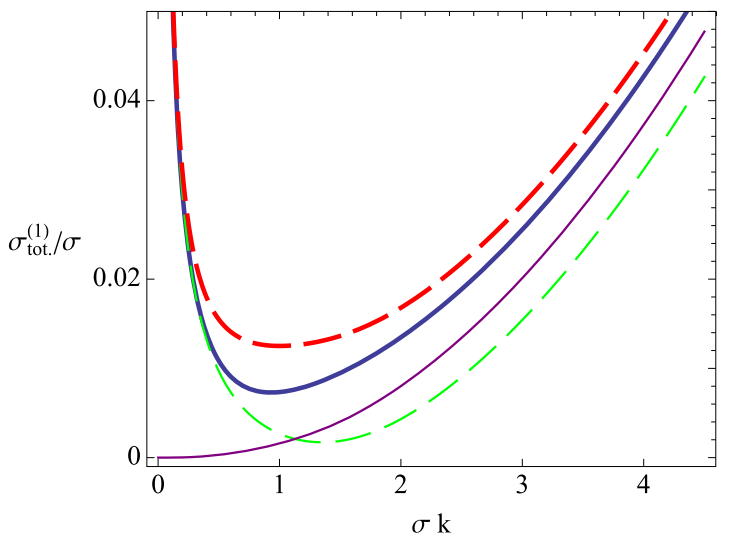

FIG. 2. Plots of $\sigma_{\text {tot. }}^{(1)} / \sigma$ as a function of $\sigma k$ for $\lambda_{1}=-\lambda_{2}=\frac{1}{2}$ (thick solid blue curve) which is obtained by the thin-layer quantization scheme [18], $\lambda_{1}=\lambda_{2}=\frac{1}{2}$ (thin dashed green curve), $\lambda_{1}-\frac{1}{2}=$ $\lambda_{2}=0$ (thin solid purple curve), and $\lambda_{2}+\frac{1}{2}=\lambda_{1}=0$ (thick dashed red curve) for a Gaussian bump (25) with $\eta=0.1$.

where $I_{n}(\mathfrak{z})$ stands for the modified Bessel function of the first kind, and

$$
\begin{aligned}
\mathfrak{p}_{0}(\mathfrak{z}):= & 64 \lambda_{2}^{2}+64 \lambda_{2}\left(2 \lambda_{1}-1\right) \mathfrak{z} \\
& +\left(16-64 \lambda_{1}+128 \lambda_{1}^{2}+16 \lambda_{1} \lambda_{2}+35 \lambda_{2}^{2}\right) \mathfrak{z}^{2} \\
& +4 \lambda_{2}\left(16 \lambda_{1}+\lambda_{2}-4\right) \mathfrak{z}^{3}+8 \lambda_{2}^{2} \mathfrak{z}^{4}, \\
\mathfrak{p}_{1}(\mathfrak{z}):= & -2\left[\left(32 \lambda_{1}^{2}+80 \lambda_{1} \lambda_{2}+11 \lambda_{2}^{2}\right) \mathfrak{z}\right. \\
& +4\left(16 \lambda_{1}^{2}+5 \lambda_{2}^{2}+6 \lambda_{1} \lambda_{2}-8 \lambda_{1}-\lambda_{2}\right) \mathfrak{z}^{2} \\
& \left.+4 \lambda_{2}\left(\lambda_{2}+8 \lambda_{1}-2\right) \mathfrak{z}^{3}+4 \lambda_{2}^{2} \mathfrak{z}^{4}\right] .
\end{aligned}
$$

Figure 2 shows the plots of the total scattering cross section (28) as a function of $\sigma k$ for different choices of the parameters $\lambda_{1}$ and $\lambda_{2}$.

\section{CONSEQUENCES OF A SMALL VIOLATION OF CYLINDRICAL SYMMETRY}

The results of the preceding section apply to surfaces with cylindrical symmetry. In this section we examine the effects of the perturbations of the surface that violate this symmetry. We quantify these by replacing (14) with

$$
z=f(r)+\epsilon \sum_{n=1}^{\infty}\left[a_{n}(r) \cos (n \theta)+b_{n}(r) \sin (n \theta)\right],
$$

where $\epsilon$ is a real perturbation parameter, $a_{n}, b_{n}:[0, \infty) \rightarrow \mathbb{R}$ are smooth functions that decay asymptotically, i.e., $\left|a_{n}(r)\right|+$ $\left|b_{n}(r)\right| \rightarrow 0$ as $r \rightarrow \infty, \theta$ is the angular polar coordinate, and we demand that for all $r \in[0, \infty)$

$$
|\epsilon| \sum_{n=0}^{\infty}\left[\left|a_{n}(r)\right|+\left|b_{n}(r)\right|\right] \ll|f(r)| .
$$

This allows us to ignore the quadratic and higher order terms in powers of $\epsilon$.

Equation (29) defines an embedded surface that we denote by $\tilde{S}$. In cylindrical coordinates $(r, \theta)$, the components of the metric $\tilde{g}$ of $\tilde{S}$ take the form

$$
\tilde{g}_{i j}=g_{i j}+\epsilon g_{\epsilon i j},
$$

where $g_{i j}$ are given by (15), and

$$
\left[g_{\epsilon i j}\right]:=\sum_{n=1}^{\infty}\left[\begin{array}{cc}
2 \dot{f}\left(\dot{a}_{n} \cos n \theta+\dot{b}_{n} \sin n \theta\right) & -n \dot{f}\left(a_{n} \sin n \theta-b_{n} \cos n \theta\right) \\
-n \dot{f}\left(a_{n} \sin n \theta-b_{n} \cos n \theta\right) & 0
\end{array}\right]
$$

Similarly, we write the corresponding Gaussian and mean curvatures as

$$
\tilde{K}=K+\epsilon K_{\epsilon}, \quad \tilde{M}=M+\epsilon M_{\epsilon},
$$

where $K$ and $M$ are given by (16), and

$$
\begin{aligned}
K_{\epsilon}:= & \sum_{n=1}^{\infty} \cos (n \theta)\left[\frac{r \dot{f} \ddot{a}_{n}-n^{2} \ddot{f} a_{n}}{r^{2}\left(1+\dot{f}^{2}\right)^{2}}+\frac{\left(1-3 \dot{f}^{2}\right) \ddot{f} \dot{a}_{n}}{r\left(1+\dot{f}^{2}\right)^{3}}\right]+\sum_{n=1}^{\infty} \sin (n \theta)\left[\frac{r \dot{f} \ddot{b}_{n}-n^{2} \ddot{f} b_{n}}{r^{2}\left(1+\dot{f}^{2}\right)^{2}}+\frac{\left(1-3 \dot{f}^{2}\right) \ddot{f} \dot{b}_{n}}{r\left(1+\dot{f}^{2}\right)^{3}}\right], \\
M_{\epsilon}:= & \sum_{n=1}^{\infty} \cos (n \theta)\left[-\frac{n^{2} a_{n}}{2 r^{2}\left(1+\dot{f}^{2}\right)^{1 / 2}}-\frac{3 \dot{f} \ddot{f} \dot{a}_{n}}{2\left(1+\dot{f}^{2}\right)^{5 / 2}}+\frac{\dot{a}_{n}+r \ddot{a}_{n}}{2 r\left(1+\dot{f}^{2}\right)^{3 / 2}}\right] \\
& +\sum_{n=1}^{\infty} \sin (n \theta)\left[-\frac{n^{2} b_{n}}{2 r^{2}\left(1+\dot{f}^{2}\right)^{1 / 2}}-\frac{3 \dot{f} \ddot{f} \dot{b}_{n}}{2\left(1+\dot{f}^{2}\right)^{5 / 2}}+\frac{\dot{b}_{n}+r \ddot{b}_{n}}{2 r\left(1+\dot{f}^{2}\right)^{3 / 2}}\right] .
\end{aligned}
$$

Recall that we require $r^{-1} \dot{f}(r)$ to tend to a finite value as $r \rightarrow 0$, so that $K$ and $M$ do not have singularities. Similarly, demanding $\tilde{K}$ and $\tilde{M}$ to be regular functions restricts the choice of $f(r), a_{n}(r)$, and $b_{n}(r)$.

We begin our analysis of the scattering of a scalar particle due to nontrivial geometry of $\tilde{S}$ by expressing the corresponding scattering amplitude $\tilde{f}^{(1)}\left(\vec{k}^{\prime}, \vec{k}\right)$ in the form

$$
\tilde{f}^{(1)}\left(\vec{k}^{\prime}, \vec{k}\right)=f^{(1)}\left(\vec{k}^{\prime}, \vec{k}\right)+\epsilon f_{\epsilon}^{(1)}\left(\vec{k}^{\prime}, \vec{k}\right),
$$


where $f^{(1)}\left(\vec{k}^{\prime}, \vec{k}\right)$ is given by (20), and $f_{\epsilon}^{(1)}(\vec{k}, \vec{k})$ describes the effects of the violation of cylindrical symmetry. To compute the latter, we employ (12) and the identities

$$
\begin{aligned}
& \int_{0}^{2 \pi} e^{i x \cos \theta} \cos (n \theta-\varphi) d \theta=2 i^{n} \pi J_{n}(x) \cos \varphi, \\
& \int_{0}^{2 \pi} e^{i x \cos \theta} \sin (n \theta-\varphi) d \theta=-2 i^{n} \pi J_{n}(x) \sin \varphi,
\end{aligned}
$$

which hold for real variables $x$ and $\varphi$. The result of this calculation is

$$
\begin{aligned}
f_{\epsilon}^{(1)}\left(\vec{k}^{\prime}, \vec{k}\right) & =\sqrt{\frac{\pi}{8 k}} e^{-3 \pi i / 4} \sum_{\substack{n=-\infty \\
n \neq 0}}^{\infty} \int_{0}^{\infty} d r i^{n}\left(k^{2}\left\{\cos \Theta \mathcal{X}\left[a_{|n|}(r)\right]+\frac{n}{|n|} \sin \Theta \mathcal{X}\left[b_{|n|}(r)\right]\right\} J_{2+n}[2 k r \sin (\Theta / 2)]\right. \\
& +k\left\{\sin (\Theta / 2) \mathcal{Y}\left[a_{|n|}(r)\right]-\frac{n}{|n|} \cos (\Theta / 2) \mathcal{Y}\left[b_{|n|}(r)\right]\right\} J_{1+n}[2 k r \sin (\Theta / 2)] \\
& \left.+r\left[2 \lambda_{1} K_{|n|}^{(a)}+4 \lambda_{2} M M_{|n|}^{(a)}-k^{2} \frac{\dot{f} \dot{a}_{|n|}}{(1+\dot{f})^{2}}\right] J_{n}[2 k r \sin (\Theta / 2)]\right),
\end{aligned}
$$

where $\mathcal{X}$ and $\mathcal{Y}$ are differential operators that act on smooth test functions $\phi(r)$ according to

$$
\begin{aligned}
\mathcal{X}[\phi(r)] & :=\frac{n \dot{f} \phi}{1+\dot{f}^{2}}-\frac{r \dot{f} \dot{\phi}}{\left(1+\dot{f}^{2}\right)^{2}}, \\
\mathcal{Y}[\phi(r)]: & =\frac{n(n+1) \dot{f} \phi}{r\left(1+\dot{f}^{2}\right)}+\frac{n \ddot{f} \phi-2 \dot{f} \dot{\phi}-r \dot{f} \ddot{\phi}}{\left(1+\dot{f}^{2}\right)^{2}}-\frac{r \ddot{f}\left(1-3 \dot{f}^{2}\right) \dot{\phi}}{\left(1+\dot{f}^{2}\right)^{3}}
\end{aligned}
$$

and

$$
\begin{aligned}
K_{n}^{(a)} & :=\frac{r \dot{f} \ddot{a_{n}}-n^{2} \ddot{f} a_{n}}{r^{2}\left(1+\dot{f}^{2}\right)^{2}}+\frac{\left(1-3 \dot{f}^{2}\right) \ddot{f} \dot{a}_{n}}{r\left(1+\dot{f}^{2}\right)^{3}}, \\
M_{n}^{(a)} & :=-\frac{n^{2} a_{n}}{2 r^{2}\left(1+\dot{f}^{2}\right)^{1 / 2}}-\frac{3 \dot{f} \ddot{f} \dot{a}_{n}}{2\left(1+\dot{f}^{2}\right)^{5 / 2}}+\frac{\dot{a}_{n}+r \ddot{a}_{n}}{2 r\left(1+\dot{f}^{2}\right)^{3 / 2}} .
\end{aligned}
$$

For example, consider the perturbed Gaussian bump given by the following choice for the functions $f, a_{n}$, and $b_{n}$ :

$$
\begin{gathered}
f(r)=\delta e^{-r^{2} / 2 \sigma^{2}}, \quad a_{1}(r)=\frac{r}{\alpha_{1}} f(r), \quad a_{2}(r)=\frac{r^{2}}{\alpha_{2}^{2}} f(r), \\
b_{1}(r)=\frac{r}{\beta_{1}} f(r), \quad b_{2}(r)=\frac{r^{2}}{\beta_{2}^{2}} f(r), \quad a_{n}(r)=b_{n}(r)=0, \text { for } n \geqslant 2,
\end{gathered}
$$

where $\alpha_{1}, \alpha_{2}, \beta_{1}$, and $\beta_{2}$ are constant parameters with the dimension of length. Then $f^{(1)}\left(\vec{k}^{\prime}, \vec{k}\right)$ is given by (26), and we can evaluate the integral on the right-hand side of (33) to find

$$
\begin{aligned}
\tilde{f}_{\epsilon}^{(1)}\left(\vec{k}^{\prime}, \vec{k}\right)= & \sqrt{\frac{\pi}{2 k}} e^{-3 \pi i / 4}\left\{-\frac{\sigma^{4} k^{2} \sin ^{2} \frac{\Theta}{2}}{2 \alpha_{2}^{2}}\left[\csc ^{2} \frac{\Theta}{2}-\sigma^{2} k^{2}-4 \lambda_{1}\left(1-\sigma^{2} k^{2} \sin ^{2} \frac{\Theta}{2}\right)+\lambda_{2} \sigma^{4} k^{4} \sin ^{4} \frac{\Theta}{2}\right]\right. \\
& \left.+\frac{i \sigma^{2} k \sin \left(\frac{\Theta}{2}\right)}{2 \alpha_{1}}\left[-\sigma^{2} k^{2}+4 \lambda_{1} \sigma^{2} k^{2} \sin ^{2} \frac{\Theta}{2}+\lambda_{2}\left(2+\sigma^{4} k^{4} \sin ^{4} \frac{\Theta}{2}\right)\right]\right\} \exp \left(-\sigma^{2} k^{2} \sin ^{2} \frac{\Theta}{2}\right) \eta+O\left(\eta^{2}\right) .
\end{aligned}
$$

Substituting (26) and (36) in (32), we can express the scattering amplitude for the surface $\tilde{S}$ as

$$
\tilde{f}^{(1)}\left(\vec{k}^{\prime}, \vec{k}\right)=f^{(1)}\left(\vec{k}^{\prime}, \vec{k}\right)\left\{1+\epsilon\left[\mathcal{Z}_{1}\left(\vec{k}^{\prime}, \vec{k}\right)+i \mathcal{Z}_{2}\left(\vec{k}^{\prime}, \vec{k}\right)\right]\right\},
$$

where

$$
\begin{gathered}
\mathcal{Z}_{1}\left(\vec{k}^{\prime}, \vec{k}\right):=\frac{2 \sigma^{2}}{\alpha_{2}^{2}}\left[1-\sigma^{2} k^{2} \sin ^{2} \frac{\Theta}{2}-\frac{\lambda_{2}\left[\left(1-\sigma^{2} k^{2} \sin ^{2} \frac{\Theta}{2}\right)^{2}+1\right]}{\left(4 \lambda_{1} \sin ^{2} \frac{\Theta}{2}-1\right) \sigma^{2} k^{2}+\lambda_{2}\left(\sigma^{4} k^{4} \sin ^{4} \frac{\Theta}{2}+2\right)}\right] \\
\mathcal{Z}_{2}\left(\vec{k}^{\prime}, \vec{k}\right):=\frac{2 \sigma^{2} k \sin \frac{\Theta}{2}}{\alpha_{1}}
\end{gathered}
$$




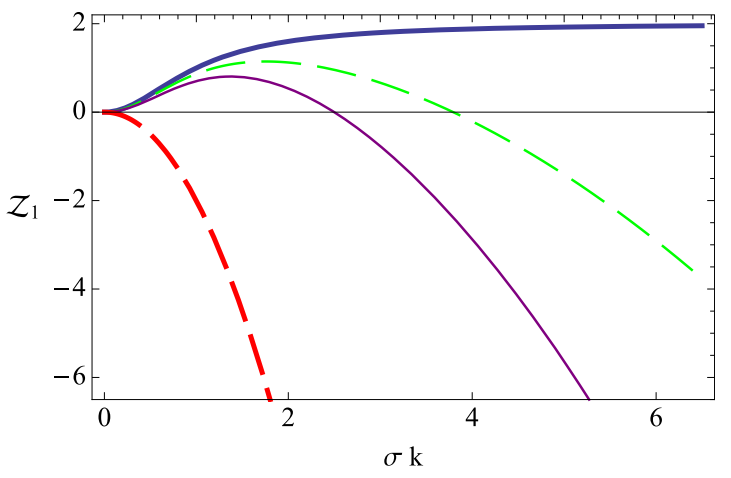

FIG. 3. Plots of $\mathcal{Z}_{1}$ as a function of $\sigma k$ for $\alpha_{2}=\sigma$ and $\Theta=0$, i.e., forward scattering (thick solid blue curve), $\Theta=\pi / 6$ (thin dashed green curve), and $\Theta=\pi / 4$ (thin solid purple curve), and $\Theta=\pi$, i.e., backward scattering (thick dashed red curve) for a perturbed Gaussian bump (25) determined by (34) and (35).

In particular, to the leading order in $\eta$ and $\epsilon$ the differential cross section for the surface $\tilde{S}$ has the form

$$
\begin{aligned}
\frac{d \tilde{\sigma}^{(1)}\left(\vec{k}^{\prime}, \vec{k}\right)}{d \Omega} & =\left|\tilde{f}^{(1)}\left(\vec{k}^{\prime}, \vec{k}\right)\right|^{2} \\
& =\left|f^{(1)}\left(\vec{k}^{\prime}, \vec{k}\right)\right|^{2}\left[1+2 \epsilon \mathcal{Z}_{1}\left(\vec{k}^{\prime}, \vec{k}\right)\right] .
\end{aligned}
$$

According to this equation the effect of the violation of cylindrical symmetry that is given by (34) and (35) is encoded in the value of $\mathcal{Z}_{1}\left(\vec{k}^{\prime}, \vec{k}\right)$. Figure 3 shows the graph of this quantity as a function of $\sigma k$ for $\lambda_{1}=-\lambda_{2}=1 / 2$, which follow from thin-layer quantization prescription, $\alpha_{2}=\sigma$, and $\Theta=0, \pi / 6, \pi / 4$, and $\pi$. As seen from this figure we can consistently apply (40) for values of $k$ that are of the order of $\sigma^{-1}$ or smaller. In particular, for $\sigma \epsilon / \alpha_{1} \ll 1$ and $\sigma \epsilon / \alpha_{2} \ll 1$, we can ignore this kind of violations of cylindrical symmetry. Notice also that for large values of $k$, the violation of cylindrical symmetry does not affect the forward scattering cross section. This is not the case for a nonforward scattering cross section.

\section{GEOMETRIC SCATTERING FOR A SURFACE WITH LOCAL CYLINDRICAL SYMMETRY}

Consider an embedded surface $\mathcal{S}$ with local cylindrical symmetry that is given by

$$
z=\sum_{j=1}^{N} f_{j}\left(\left|\vec{x}-\overrightarrow{\mathfrak{c}}_{j}\right|\right)
$$

where $N$ is a positive integer, $f_{j}:[0, \infty) \rightarrow \mathbb{R}$ are smooth functions such that $\lim _{r \rightarrow 0} \dot{f}_{j}(r) / r$ exists, and $\overrightarrow{\mathfrak{c}}_{j}=\left(a_{j}, b_{j}\right)$ are centers of local cylindrical symmetry.

Suppose that the functions $f_{j}$ decay sufficiently fast away from 0 so that for each $j$ we can approximate $f_{j}\left(\left|\vec{r}-\overrightarrow{\mathfrak{c}}_{j}\right|\right)$ by a function that vanishes outside a disk $\mathscr{D}_{j}$ centered at $\mathfrak{c}_{j}$ with $\mathscr{D}_{j^{\prime}} \cap \mathscr{D}_{j}=\varnothing$ for $j^{\prime} \neq j$. If we use the first Born approximation to determine the geometric scattering properties of such a surface, the scattering amplitude for $\mathcal{S}$ takes the form

$$
\mathfrak{f}^{(1)}\left(\vec{k}^{\prime}, \vec{k}\right)=\sum_{j=1}^{N} \mathfrak{f}_{j}^{(1)}\left(\vec{k}^{\prime}, \vec{k}\right)
$$

where $\mathfrak{f}_{j}^{(1)}\left(\vec{k}^{\prime}, \vec{k}\right)$ stands for the scattering amplitude associated with the surface $S_{j}$ given by

$$
z=f_{j}\left(\left|\vec{r}-\overrightarrow{\mathfrak{c}}_{j}\right|\right) .
$$

We can obtain $S_{j}$ from a surface $S_{0 j}$ with cylindrical symmetry about the $z$ axis by a simple space translation. It is not difficult to show that $\mathfrak{f}_{j}^{(1)}\left(\vec{k}^{\prime}, \vec{k}\right)$ is related to the scattering amplitude $f_{0 j}^{(1)}\left(\vec{k}^{\prime}, \vec{k}\right)$ of $S_{0 j}$ according to

$$
\mathfrak{f}_{j}^{(1)}\left(\vec{k}^{\prime}, \vec{k}\right)=e^{i\left(\vec{k}-\vec{k}^{\prime}\right) \cdot \mathfrak{c}_{j}} f_{0 j}^{(1)}\left(\vec{k}^{\prime}, \vec{k}\right) .
$$

In view of (42) and (44), we can use the results of Sec. III to compute the scattering amplitude of $\mathcal{S}$. This is particularly easy when $f_{j}$ [and consequently $f_{0 j}^{(1)}\left(\vec{k}^{\prime}, \vec{k}\right)$ ] coincide. In this case,

$$
\mathfrak{f}^{(1)}\left(\vec{k}^{\prime}, \vec{k}\right)=C\left(\vec{k}^{\prime}, \vec{k}\right) f^{(1)}\left(\vec{k}^{\prime}, \vec{k}\right)
$$

where

$$
C\left(\vec{k}^{\prime}, \vec{k}\right):=\sum_{j=1}^{N} e^{i\left(\vec{k}-\vec{k}^{\prime}\right) \cdot \mathfrak{c}_{j}}
$$

and $f^{(1)}\left(\vec{k}^{\prime}, \vec{k}\right)$ is the common value of $f_{0 j}^{(1)}\left(\vec{k}^{\prime}, \vec{k}\right)$.

If $\overrightarrow{\mathfrak{c}}_{j}$ form a lattice, $j$ stands for an index pair $(m, n)$ and

$$
\overrightarrow{\mathfrak{c}}_{j}=\overrightarrow{\mathfrak{c}}_{m n}=m \vec{a}+n \vec{b}
$$

where $\vec{a}$ and $\vec{b}$ are constant vectors. We can use this relation to perform the sum in (46). Supposing that $m$ and $n$, respectively, take values in the intervals $\left[m_{1}, m_{2}\right]$ and $\left[n_{1}, n_{2}\right]$, substituting (47) in (46), and using the identity

$$
\sum_{j=j_{1}}^{j_{2}} \mathfrak{z}^{j}=\frac{\mathfrak{z}^{j_{2}+1}-\mathfrak{z}^{j_{1}}}{\mathfrak{z}-1}
$$

we obtain

$$
C\left(\vec{k}^{\prime}, \vec{k}\right)=\frac{\left(e^{i\left(m_{2}+1\right) k_{a}}-e^{i m_{1} k_{a}}\right)\left(e^{i\left(n_{2}+1\right) k_{b}}-e^{i n_{1} k_{a}}\right)}{\left(e^{i k_{a}}-1\right)\left(e^{i k_{b}}-1\right)},
$$

where

$$
k_{a}:=\left(\vec{k}-\vec{k}^{\prime}\right) \cdot \vec{a}, \quad k_{b}:=\left(\vec{k}-\vec{k}^{\prime}\right) \cdot \vec{b} .
$$

A simple example is a finite lattice of Gaussian bumps, such as those forming a liquid-helium Wigner lattice:

$$
z=\delta \sum_{m=m_{1}}^{m_{2}} \sum_{n=n_{1}}^{n_{2}} e^{-\left(\vec{r}-\overrightarrow{\mathfrak{c}}_{m n}\right)^{2} / 2 \sigma^{2}},
$$

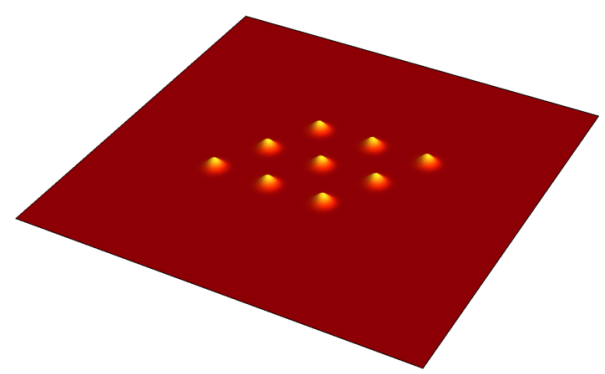

FIG. 4. Schematic view of a surface involving the triangular lattice of Gaussian bumps given by (51) and (52). 

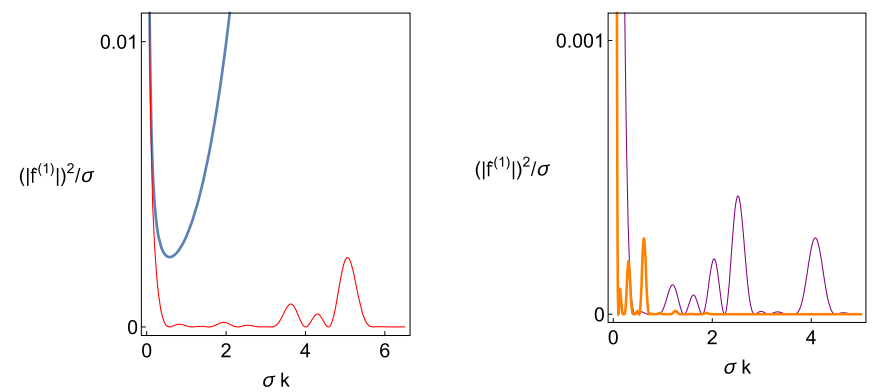

FIG. 5. Plots of $\left|f^{(1)}\left(\vec{k}^{\prime}, \vec{k}\right)\right|^{2} / \sigma$ as a function of $\sigma k$ for the surface involving the triangular lattice of Gaussian bumps given by (47), (50), (51), and (52), $a=10 \sigma, \eta=0.01, \lambda_{1}=-\lambda_{2}=1 / 2$, and $\Theta=0$ (left panel, thick blue curve), $\Theta=\pi / 6$ (left panel, thin red curve), $\Theta=$ $\pi / 4$ (right panel, thin purple curve), and $\Theta=\pi$ (right panel, thick orange curve).

where $\left|\mathfrak{c}_{m^{\prime} n^{\prime}}-\mathfrak{c}_{m n}\right| \gg \sigma$ for $\left(m^{\prime}, n^{\prime}\right) \neq(m, n)$. For this surface, the geometric scattering amplitude has the form (45) with $C\left(\vec{k}^{\prime}, \vec{k}\right)$ and $f^{(1)}\left(\vec{k}^{\prime}, \vec{k}\right)$, respectively, given by (48) and (26). Notice, however, that in order for the quadratic and higher order terms in $\eta$ on the right-hand side of (26) to be negligible, we should have $\left(m_{2}-m_{1}\right)\left(n_{2}-n_{1}\right) \eta \ll 1$.

To be specific, consider taking

$$
\begin{aligned}
& \vec{a}=a(1,0), \quad \vec{b}=a\left(\frac{1}{2}, \frac{\sqrt{3}}{2}\right), \\
& m_{1}=n_{1}=-1, \quad m_{2}=n_{2}=1,
\end{aligned}
$$

where $a$ is the lattice constant. This corresponds to a triangular lattice consisting of nine Gaussian bumps, as depicted in Fig. 4. In a coordinate system in which $\vec{k}$ is along the $x$ axis, we have $\vec{k}^{\prime}=k(\cos \Theta, \sin \Theta)$. This together with (51) imply

$$
k_{a}=a k(1-\cos \Theta), \quad k_{b}=\frac{a k}{2}(1-\cos \Theta-\sqrt{3} \sin \Theta) .
$$

Substituting (52) and (53) in (48) and using the result together with (26) and (45) we can derive an analytic expression for the geometric scattering amplitude of the surface defined by (50). Figure 5 shows the graph of differential cross section $\left|\mathfrak{f}^{(1)}\left(\vec{k}^{\prime}, \vec{k}\right)\right|^{2}$ as a function of $\sigma k$ for $a=10 \sigma, \eta=0.01, \lambda_{1}=$ $-\lambda_{2}=1 / 2$, and different values of $\Theta$.

\section{SUMMARY AND CONCLUDING REMARKS}

A classical free particle that is constrained to move on an embedded surface feels the effect of the nontrivial geometry of the surface through its contribution to the kinetic energy term in the Hamiltonian. For a quantum particle there can be an additional contribution to the Hamiltonian that arises in the form of a quantum mechanical geometric potential involving both the Gaussian and mean curvatures of the surface. The strength of this curvature interaction is determined by a pair of coupling constants whose value cannot be determined from the first principles. For a specific system these constants enter in the associated physical quantities.

In this article we have considered a nonrelativistic spinless free particle moving on an asymptotically flat embedded surface and examined its scattering due to the nontrivial geometry of this surface. In particular, we have used the first Born approximation to calculate the geometric scattering amplitude for a surface with global cylindrical symmetry and examined the effects of perturbations of the surface that violate this symmetry. We have also extended our analysis to surfaces with local cylindrical symmetry. This allows for an analytic treatment of surfaces formed by a finite lattice of well-separated bumps. Our results reveal the possibility of determining the values of the unknown curvature coefficients using the scattering data.

For a cylindrically symmetric surfaces, only the mean curvature contributes to the forward scattering amplitude. This is not the case for the backward scattering amplitude, which receives contributions from both the mean and Gaussian curvatures. In view of this observation, one can in principle determine the values of the unknown curvature constants using only the forward and backward scattering data. Therefore if it turns out that both $\lambda_{1}$ and $\lambda_{2}$ take nonzero values, then an experimental realization of our setup would provide means for independent measurements of the physical effects of intrinsic and extrinsic geometry of the surface.

Once the curvature coefficients are determined we can use our analytical results to make predictions on the behavior of the geometric scattering cross section and its dependence on the shape of the surface. For example, if the forward and backward scattering data for a Gaussian bump confirm the choice given by the thin-layer quantization scheme, i.e., $\lambda_{1}=-\lambda_{2}=1 / 2$, we expect the differential cross section for nonforward scattering to attain a single peak as we vary the wave number of the incident wave. For a lattice of Gaussian bumps the differential cross section develops several peaks.

\section{ACKNOWLEDGMENTS}

This work has been supported by the Scientific and Technological Research Council of Turkey (TÜBITAK) in the framework of Project No. 117F108 and by the Turkish Academy of Sciences (TÜBA).
[1] B. S. DeWitt, Phys. Rev. 85, 653 (1952).

[2] B. S. DeWitt, Rev. Mod. Phys. 29, 377 (1957).

[3] K. S. Cheng, J. Math. Phys. 13, 1723 (1972).

[4] G. A. Ringwood, J. Phys. A 9, 1253 (1976).

[5] M. K. Ali, Can. J. Phys. 74, 255 (1992).

[6] B. S. DeWitt, Supermanifolds (Cambridge University Press, Cambridge, 1992).
[7] A. Foerster, H. O. Girotti, and P. S. Kuhn, Phys. Lett. A 195, 301 (1994).

[8] A. Mostafazadeh, J. Math. Phys. 35, 1095 (1994).

[9] A. Mostafazadeh, J. Math. Phys. 35, 1125 (1994).

[10] R. Penrose, Proc. R. Soc. London A 284, 159 (1965).

[11] M. P. Ryan Jr. and A. V. Turbiner, Phys. Lett. A 333, 30 (2004). 
[12] C. DeWitt-Morette, K. D. Elworthy, B. L. Nelson, and G. S. Sammelman, Ann. Inst. Henry Poincaré A 32, 327 (1980).

[13] H. Kleinert, Phys. Lett. B 236, 315 (1990).

[14] M. S. Marinov, Phys. Rep. 60, 1 (1980).

[15] A. Mostafazadeh, Phys. Rev. A 54, 1165 (1996).

[16] P. A. M. Dirac, Lectures on Quantum Mechanics (Yeshiva University Press, New York, 1964).

[17] H. Jensen and H. Koppe, Ann. Phys. (NY) 63, 586 (1971).

[18] R. C. T. da Costa, Phys. Rev. A 23, 1982 (1981).

[19] J. Tolar, in Group Theoretical Methods in Physics, Proceedings of the XVI International Colloquium Held at Varna, Bullgaria, June 15-20, Lect. Notes in Phys. Vol. 313, edited by H.-D. Doebner, J.-D. Hennig, and T. D. Palev (Springer, Berlin, 1988), pp. 268-274.

[20] P. Maraner, J. Phys. A 28, 2939 (1995).

[21] N. Ogawa, Prog. Theor. Phys. 87, 513 (1992).

[22] R. Froese and I. Herbst, Commun. Math. Phys. 220, 489 (2001).

[23] P. C. Schuster and R. L. Jaffe, Ann. Phys. 307, 132 (2003).

[24] D. Krejčiř́ík, N. Raymond, and M. Tušek, J. Geom. Anal. 25, 2546 (2015).
[25] A. V. Golovnev, Rep. Math. Phys. 64, 59 (2009).

[26] D. M. Xun and Q. H. Liu, Ann. Phys. (NY) 341, 132 (2014).

[27] M. P. Do Carmo, Differential Geometry of Curves and Surfaces (Prentice-Hall, Upper Saddle River, NJ, 1976).

[28] N. Ogawa, K. Fujii, N. Chepiko, and A. Kobushkin, Prog. Theor. Phys. 85, 1189 (1991).

[29] L. Kaplan, N. T. Maitra, and E. J. Heller, Phys. Rev. A 56, 2592 (1997).

[30] J. Onoe, T. Ito, H. Shima, H. Yoshioka, and S.-I. Kimura, Europhys. Lett. 98, 27001 (2012).

[31] V. Atanasov and R. Dandoloff, Phys. Lett. A 371, 118 (2007).

[32] C. Ortix, S. Kiravittaya, O. G. Schmidt, and J. van den Brink, Phys. Rev. B 84, 045438 (2011).

[33] K. V. R. A. Silva, C. F. de Freitas, and C. Filgueiras, Eur. Phys. J. B 86, 147 (2013).

[34] V. Vadakkumbatt, E. Joseph, A. Pal, and A. Ghosh, Nat. Commun. 5, 4571 (2014).

[35] H. Pahlavani and M. Botchekananfard, Physca B 459, 88 (2015).

[36] J. J. Sakurai, Modern Quantum Mechanics (Addison-Wesley, New York, 1985). 